\title{
A comparative study of serological diagnosis of Dengue outbreak 2019
}

\author{
Muhammad Bilal Habib ${ }^{1}$, Noreen Sher Akbar ${ }^{2}$, Anber Saleem ${ }^{3}$
}

1. National Institute of Health Islamabad Pakistan 44000.

2. DBS\&H CEME National University of Sciences and Technology, Islamabad, Pakistan.

3. School of Dentistry, Shaheed zulfiqar ali Bhutto medical university Islamabad.

\begin{abstract}
Purpose: The interpretation \& correlation of the different laboratory parameters in positive dengue cases in order to evaluate that which laboratory test is more significant for diagnosis of Dengue.

Methods: Prospective examination of samples (patients' serum) for dengue virus of different genotype by using multiplex anti-dengue IgM, IgG. We have done NS-1 test by (ICT) immunochromatographic devices, and complete blood picture (CBC) by Sysmex XP-100.

Result: Detection of Viral RNA in 100 patients showed effects in the total of 73 (73.0\%) samples. This graphical comparison shows the whole positive cases including dengue NS-I antigen, dengue serology (IgM \& IgG), total 62 positive cases of NS-I are detected, 10 positive cases of dengue IgM and 9 positive cases of IgG detected, in which Complete Blood Test (CBC) shows remarkable reduction in Platelets (32 cases) and Leucopenia in (24 positive cases).

Conclusion: In this research, it is concluded that the diagnosis of dengue cases is preliminary limited to initial stages i.e. CBC or sometimes dengue NS-I, as dengue IgM severity is more effective than that of Dengue NS-I \& IgG. Many patients who had negative results in CBC and NS-1 testing, became positive when IgM and IgG serology testing has been done.

Keywords: Dengue; NS-1; IgG; IgM; Immunochrometographic test.

DOI: https://dx.doi.org/10.4314/ahs.v21i3.20

Cite as: Habib MB, Akbar NS, Saleem A. A comparative study of serological diagnosis of Dengue outbreak 2019. Afri Health Sci. 2021;21(3). 1117-1123. https:// dx.doi.org/10.4314/abs.v21i3.20
\end{abstract}

\section{Background}

Mosquito born disease commonly known as Dengue mainly caused by viruses which are serologically interrelated but antigenically diverse single stranded positive RNA virus. Genus Flavivirus (Family Flaviviridae) has four serotypes which are DENV-I, DENV-II, DENVIII \& DENV-IV. Dengue virus lives in the clean environment therefore people living in clean environment are mostly prone to infections. Dengue Virus is most common in geographically distribution of Asian Countries ${ }^{1}$. There is foremost part of ecological factors like temperature for the survival of adult vector. In effective periods these strains require optimum temperature. Indication of the situation is the most infectious disease in world's major health problem list distressing tropical \& subtropical areas. This Arbovirus in most common in

\section{Corresponding author: \\ Muhammad Bilal Habib, \\ National Institute of Health Islamabad \\ Pakistan 44000. \\ Email: b.habib@nih.org.pk}

world's most populated areas in terms of morbidity \& mortality. According to W.H.O approximations, more than 2.5 billion people are at the risk of dengue infection ${ }^{2}$. In recent studies from District Swat of KPK, Pakistan 6376 cases were confirmed as Dengue and 23 deaths occurred in that area. Dengue Virus is lethal for all over the world's population and now adays this is huge problem for Public Health Correspondence. The occurrence of dengue virus is mainly observed in endemic areas especially in tropical and sub-tropical regions. According to a research, approximately 50 million people infected annually in more than 100 countries. In worldwide distribution of this infection the observed regions include Asia, America, Middle East \& Africa. The severity of infection depends upon the environmental factors of that region ${ }^{3}$.

In case of overall population, the Dengue Virus comes repeatedly per annum with more effectiveness once again as reported by W.H.O ${ }^{4}$. It is widely observed in American states that there are DENV-2 \& DENV-3 genotypes which were found more commonly and have known to be less virulent than genotypes of the Asia having same serotype. As showed by compact growth in 
both mosquitoes and culture Wang proved that DENVIII domain may play a part in viral adaptation to undeveloped hosts, the epidemiological studies reveals that the genotypes with higher virulent effect are lashing out the virus strains in lesser epidemiological impact ${ }^{5}$. In current years, substantial consideration has focused mainly towards characterizing the scope of genetic diversity in the four dengue virus serotypes, predominantly through phylogenetic analyses and sequencing of envelope (E) gene already sequenced from strains that were sampled globally ${ }^{6}$. Four serological types of dengue virus are DEN I, II, III and IV. It causes different primary and secondary infections?

There are three main markers for the detection of Dengue Virus i.e. Dengue NS-1 ICT/ ELISA, Dengue Serology $(\operatorname{IgM} \& \operatorname{IgG})$. In an acute infection with the early symptoms, IgM levels may increase which may occur within 3-5 days and persists 30-60 days after the infection exposure. IgG levels may also be raised in chronic infection type after 10 to 14 days of the infection and it will be detectable throughout life span. The technique which is used, called Hem agglutination Inhibition Assay ${ }^{8}$. There are two main vectors responsible for the dengue infections first are Aedes aegypti and second are Aedes albopictus. In this form, $A$. aegypti is less active vector, although it is responsible for the infections in endemic regions. In advanced diagnosis, PCR plays an important role in specific detection of dengue (RNA) virus ${ }^{9}$. Most of researchers concluded that very small part of infected patients may have immunoglobins $\mathrm{M}$ $(\operatorname{IgM})$ in first 3 to 4 days after onset of symptoms. The nature of outbreaks may be advanced or worst for immune-compromised persons depending upon the effectiveness of the serotypes \& strains of dengue virus ${ }^{10}$. The main root cause of the dengue virus is Aedes aegypti. The morbidity $\&$ mortality rates and economic costs are affected by the increasing incidence of dengue infection each year and still there is a raised number found from all over the world ${ }^{11}$.

The main root cause of this fatal infection is DENV. Genus Flavivirus, family: Flaviviridae. The Dengue Virus is currently known as Mosquito-borne Flavivirus because of its recurrent infections such as yellow fever, dengue fever, dengue hemorrhagic fever $\&$ dengue shock syndrome which recorded in last decade. The specific dengue virus can be detected directly in sera by using PCR techniques or by cell culture supernatants detection in infected mosquito larvae ${ }^{12}$. As it is observed that dengue is broad spectrum disease sometimes it reveals unrecognized signs \& symptoms which may be misdiagnosed co-relating with the clinical presentations of the patient i.e. fever causes may mislead to another causative agents. There is variation in incubation period as in some patients there is sudden onset of symptoms and patient may suffer many early complications i.e. myalgia, anorexia, sore throat, headache and often macular skin rash ${ }^{13}$. Most people experience self-limiting clinical course which may not lead them to severity of the infection like DHF \& DSS. Unfortunately, there is no any presence of dengue vaccine or any specific antiviral therapy all over the world. The need of the hour is to discover the antidote. Due to the recent deprived disease surveillance, there is low reporting level, low case casualty rate, complications in diagnosis, and impulsive comparative analyses, impact of dengue and true incidence is expressively higher than which is currently reported apart ${ }^{14}$. It is widely observed that dengue virus also infects the people like international travelers, physicians and doctors in western countries are most provoked with travel acquired dengue infections. Recently this infection is declared as the most important Arboviral illness in human beings. More than 100 tropic and sub-tropic countries are endemic in infection ${ }^{15}$. This study is aimed on diagnostic evaluation, to interpret \& correlate the clinico-hematological parameters in positive dengue cases.

\section{Materials and methods}

Total number of 100 blood samples is collected from patients. Following are the materials and methods which are used in diagnosis of dengue fever on the basis of clinical signs \& symptoms;

1. Complete Blood Count

2. Dengue NS-1 Antigen

3. Dengue Serology (IgM, IgG)

\section{Complete Blood Count}

CBC is performed on fully automated hematology analyzer SYSMEX XP-100 series. It is state of the art machine from Japan which gives 21 parameters.

\section{Requirements}

Sysmex XP-100, Microscope, Microscope Slides, Oil Immersion, Stains, Applicator Stick, Rotator.

\section{Procedure}

Collect the specimen by vein-cut procedure in characterized anticoagulant vacutainer. Rotate the sample on rotator for 10 minutes. Suction the sample by the test and let it investigate. Spot a perfect $3 \times 1$-inch glass slide on a level surface (stationary slide), one with a frosted end. Blend the blood well. With a fine spreader, move 
a drop of blood around 2 to $3 \mathrm{~mm}$ in distance across to the stationary slide about $1 / 4$-inch from the end of the frosted on a similar side as the composing hand. Hold the end of the stationary slide inverse the blood drop with the non-composing hand. Position a spreader (non-frosted) slide at a 25 to 30-degree point to the stationary slide and bring it once more into the drop of blood. Permit the blood to spread along the rear of the spreader slide. Label the slide at the frosted end with the Patient recognizable proof number and date, utilizing a pencil or a precious stone pen. Air-dry the blood quickly however completely (a few minutes) before recoloring. You can do this physically or with a cool air blower. Stain the slide utilizing document recoloring. Leave for 10 minutes and wash the smear with faucet water and permit the smear to air dry. Perform smear examination and report the outcomes appropriately.

\section{Dengue NS-1 Antigen}

The Dengue NS-1 Antigen test is a single step immunochromatographic test (ICT) for the rapid diagnosis of Dengue infection NS1 antigen in human serum, plasma or Whole blood. This test is advised as a guide in diagnosis of early dengue infection.

\section{Requirements}

Patient's Serum, ICT Device, Buffer, Dropper/Pipette, Stopwatch

\section{Procedure}

Bring all test devices, reagents and samples to room temperature for 15 minutes prior to performing the as- say. Use a fresh test device for every sample. The device is not reusable. Just prior to use, remove the required number of Dengue NS1 test devices from their wrapper and place them on a flat surface area. Label the test unit with the patient name or identification number. Take $100 \mu \mathrm{l}$ (3 drops) of Whole blood or serum/plasma with the included disposable pipette or a micropipette and add into the sample well. Interpret test results at 15 20 minutes. Do not interpret the test results after 20 minutes, this can give false results.

\section{Dengue IgM}

Dengue IgM is advised for in-vitro qualitative detection of Dengue IgM Antibodies in human serum or plasma and is used as a screening test for testing of collected blood samples suspected for Dengue. The kit detects all four subtypes; DEN1, DEN2, DEN3 \& DEN4 of Dengue Virus.

\section{Dengue IgG}

Dengue $\operatorname{Ig} G$ is designed for in-vitro qualitative detection of Dengue IgM Antibodies in human serum or plasma and is used as a screening test for testing of collected blood samples suspected for Dengue.

\section{Results}

\section{Clinical Findings}

Total 100 samples were investigated through diagnostics test (Dengue NS-1, Dengue Serology IgM \& IgG) which shows $77 \%$ positive patients with NS-I, $12 \%$ positive cases with Dengue IgM and $11 \%$ Dengue IgG cases were detected as showed in Figure 1.

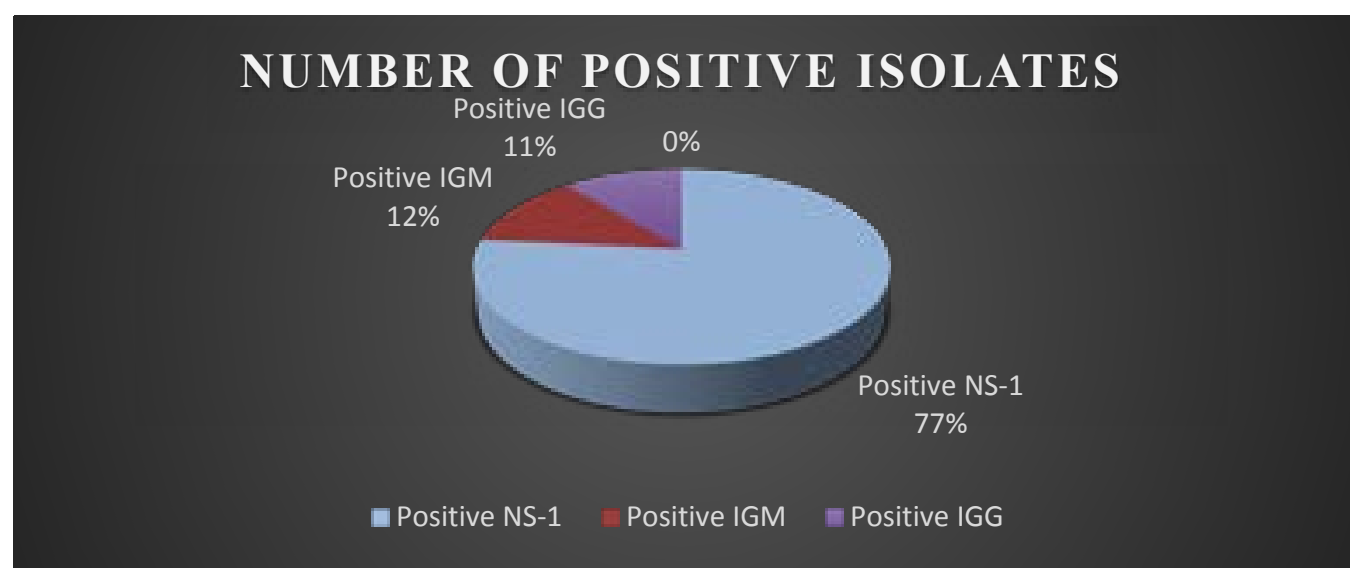

Figure 1: Total Number of Positive Isolates

Figure 2 showed the gender wise distribution of total number of samples. In which the ration of dengue pos- itive cases in (59\%) and total ration of positive (cases is $41 \%$ ). 


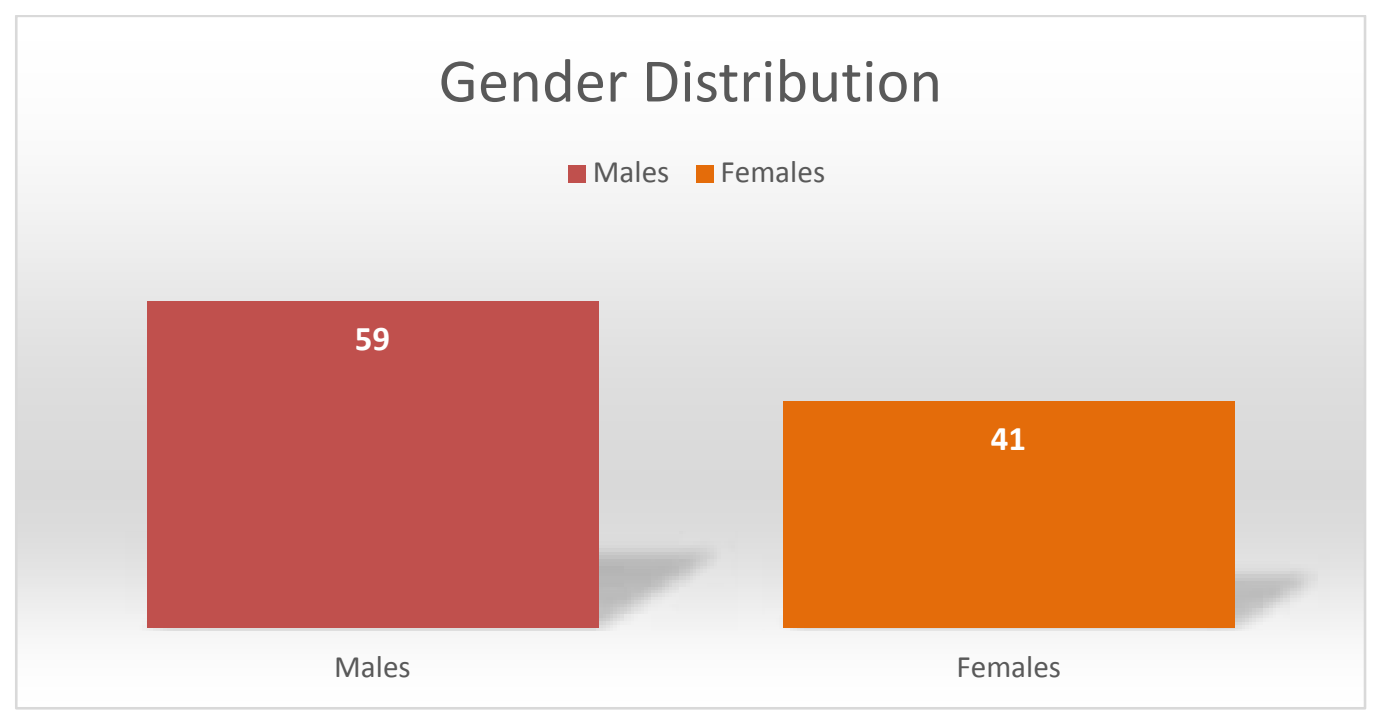

Figure 2: Gender/Sex Wise Distribution

According to the graphical distribution of all ages the ratio in 16-30 years is (25\%), the ratio in $31-45$ years showed in Figure 3. the ration in 1-15 years is (18\%), is (35\%), the ratio in $45-70$ years is $(19 \%)$ and the ratio in more than 70 years is $(3 \%)$.

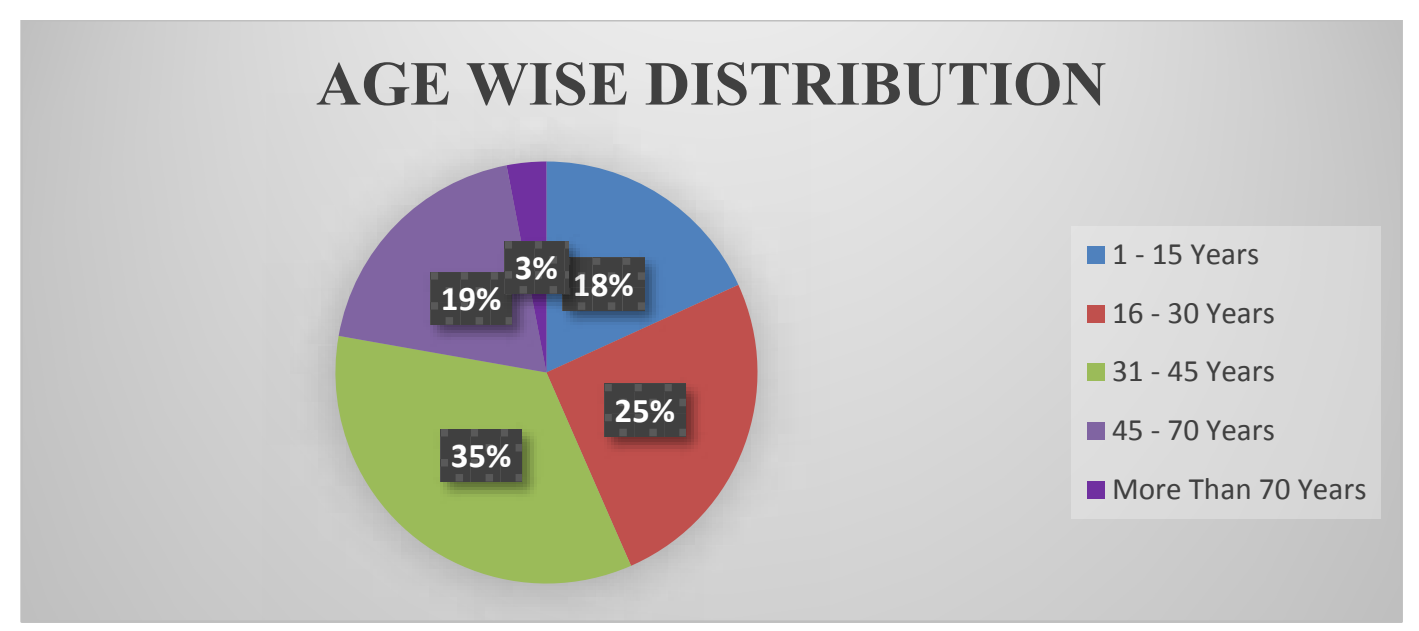

Figure 3: Age Wise Distribution

\section{Diagnostic Findings}

This graphical comparison showed in Figure 4. the whole positive cases including dengue NS-I antigen, dengue serology (IgM \& IgG), total 62 positive cases of NS-I are detected, 10 positive cases of dengue IgM and
9 positive cases of $\operatorname{IgG}$ are detected. In which Complete Blood Test (CBC) shows remarkable reduction in Platelets (32 cases) and Leucopenia in 24 positive cases which is used as diagnostic tool for the confirmation of dengue presence. 


\section{CLINICAL PARAMETERS}

\# Series 1 ESeries 2 = Series 3

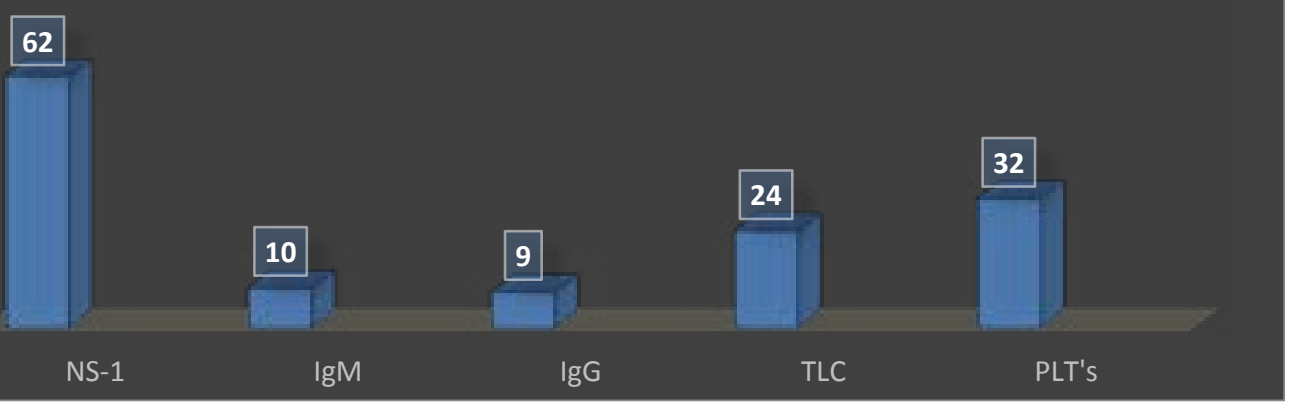

Figure 4: Clinical Comparison of Lab Parameters

We analysed the comparison of NS-1 with Dengue served that Sample number 48, 49, 54, 55, 60, and 65 IgM, the blue line upper than the cut of line shows the had negative results for NS-1, but there Dengue serolpositive cases of NS-1 and orange line upper than the ogy IgM results were positive as showed in Figure 5a, cutoff line shows the positive cases of IgM. We ob- and 5b.

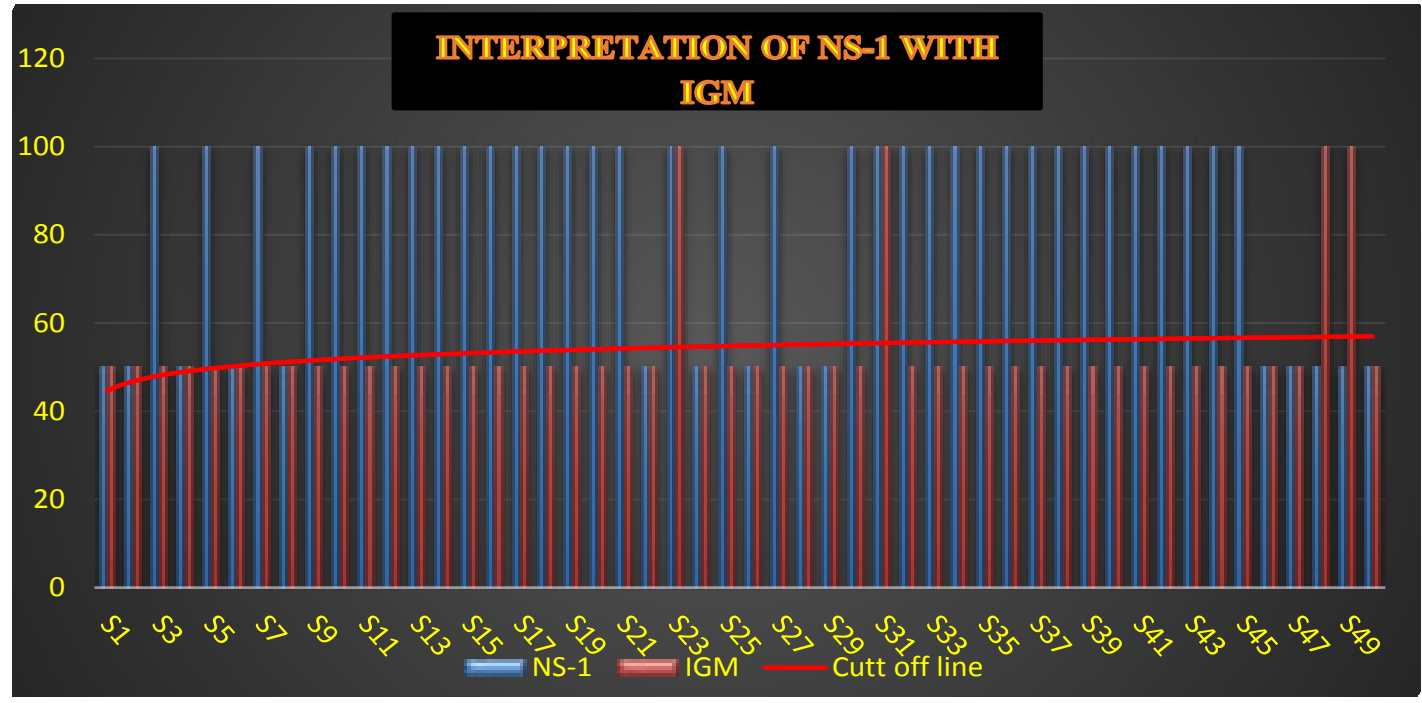

Figure 5a: Clinical Interpretation Dengue NS-1 and Dengue IgM of Total Samples (1-50)

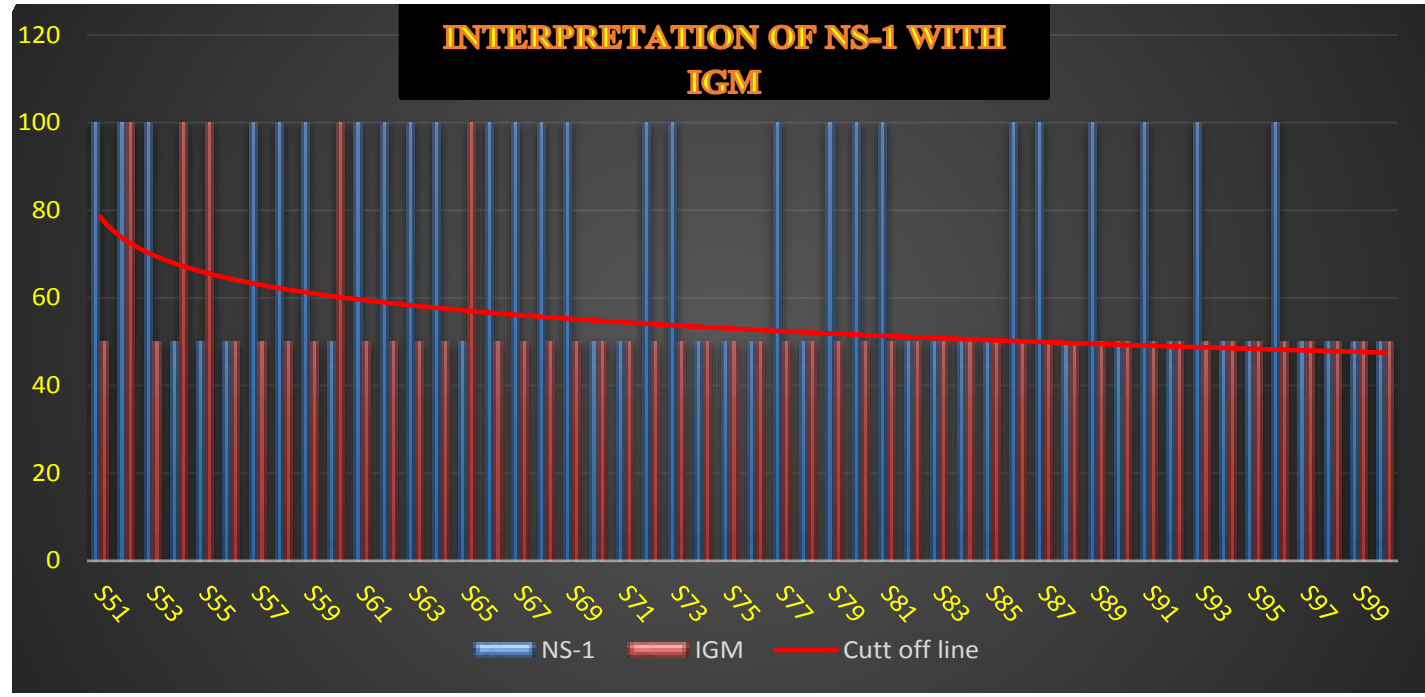

Figure 5b: Clinical Interpretation Dengue NS-1 and Dengue IgM of Total Samples (51-100) 


\section{Discussion}

In this study, the male population was greatly affected $(59 \%)$ than female $(41 \%)$ while the infection rate was less in children; same study was reported by researchers in which the prevalence in males is $(72 \%)$ which are more effective than females and children ${ }^{1}$. The highest concentrations were reported in the America in which male population is highly effected. According to this study, from all the serotypes of dengue virus DENV-I \& DENV-II serotypes are most frequently isolated. Same findings of isolated serotypes were DENV-1 and DENV-2 (90s) and DENV-2 and DENV-3 (2000$2007)^{16}$. The result of this study showed that in majority of cases blood CP were normal and NS-1 were negative but IgM and IgG of these patients became positive, another study result showed IgM levels elevated in acute infections and persists in blood for 2 months, and in chronic cases $\operatorname{IgG}$ level also raised after 14 days and detectable throughout life ${ }^{1}$. Noteworthy geographic extension has been combined with quick increments in episode cases, pestilences, and hyperendemicity, prompting the more serious types of dengue. Transmission of dengue is currently present in each World Health Organization (W.H.O) locale of the world and in excess of 125 nations are known to be dengue endemic. The genuine effect of dengue all-inclusive is hard to find out because of variables, for example, deficient sickness reconnaissance, misdiagnosis, and low degrees of detailing. Presently accessible information likely terribly thinks little of the social, monetary, and illness trouble. Assessments of the worldwide rate of dengue contaminations every year have extended between 50 million and 200 million; be that as it may, late gauges utilizing cartographic methodologies recommend this number is nearer to right around 400 million. Same relevance with another research revealed that Dengue has been present for centuries. The first recorded symptoms compatible with dengue were noted in a Chinese medical encyclopedia in $992 \mathrm{ADS}$, however originally pub $\neg$ lished by the Chin Dynasty centuries earlier (265-420 AD), prior to being formally edited ${ }^{17,18}$.

\section{Conclusion}

In this research, it is concluded that the diagnosis of dengue cases is preliminary limited to initial stages i.e. CBC or sometimes dengue NS-I, as dengue IgM severity is more effective than that of Dengue NS-I \& IgG, that why all parameters should be used for accurate diagnostic purpose of Dengue fever disease. It is humbly requested to physicians and doctors dealing with dengue outbreak every year should advance their diagnostic measures and should also pursue their prescription on complete and authenticated diagnosis of dengue patients.

\section{Abbreviations}

CBC: Complete blood count; IgM: Immunoglobulin M; IgG: Immunoglobulin G; DENV: Dengue Virus; W.H.O: World Health Organization; DHF: Dengue hemorrhagic fever;

\section{Conflict of interest}

Authors haven't any conflict of interest.

\section{Funding}

There was no funding source.

\section{Informed Consent}

There is no need of consent because no human and animal subjects were directly involved in this study.

\section{References}

1. Khan J, Khan A (2015). Incidence of dengue in 2013: dengue outbreak in District Swat, Khyber Pakhtunkhwa, Pakistan. Inter J of Fauna and Biolo Stud 2(1):1-7. 2. Reiter P (2001). Climate change and mosquito-borne disease. Environmental Health Perspectives (suppl 1):141-61. 3. Jamil B, Hasan R, Zafar A, Bewley K, Chamberlain J, Mioulet V, Rowlands M, Hewson R (2007). Dengue virus serotype 3, Karachi, Pakistan. Emerging Infectious Diseases 13(1):182.

4. Murray NE, Quam MB, Wilder-Smith A (2013). Epidemiology of dengue: past, present and future prospects. Clinical Epidemiology 5:299.

5. Gubler DJ (2012). The economic burden of dengue. The American Journal of Tropical Medicine and Hygiene 86(5):743.

6. Gubler DJ (2011). Dengue, urbanization and globalization: the unholy trinity of the 21st century. Tropical Medicine and Health 39(4SUPPLEMENT):S3-11.

7. Shepard DS, Undurraga EA, Halasa YA (2013). Economic and disease burden of dengue in Southeast Asia. PLoS Neglected Tropical Diseases 7(2).

8. Horstick O, Tozan Y, Wilder-Smith A (2015). Reviewing dengue: still a neglected tropical disease?. PLoS Neglected Tropical Diseases 9(4).

9. Bandyopadhyay B, Bhattacharyya I, Adhikary S, Konar J, Dawar N, Sarkar J, Mondal S, Singh Chauhan M, Bhattacharya N, Chakravarty A, Biswas A (2013). A comprehensive study on the 2012 dengue fever outbreak in Kolkata, India. ISRN.

10. Muller DA, Depelsenaire AC, Young PR (2017). Clin- 
ical and laboratory diagnosis of dengue virus infection. The Journal of Infectious Diseases 215(suppl_2):S89-95.

11. Sharp TM, Tomashek KM, Read JS, Margolis HS, Waterman SH (2017). A new look at an old disease: recent insights into the global epidemiology of dengue. Current Epidemiology Reports 4(1):11-21.

12. Neufeldt CJ, Cortese M, Acosta EG, Bartenschlager R (2018). Rewiring cellular networks by members of the Flaviviridae family. Nature Reviews Microbiology 16(3):125. 13. Papadakis MA, McPhee SJ (2019). Current medical diagnosis and treatment. McGraw-Hill.

14. McArthur MA, Sztein MB, Edelman R (2013). Dengue vaccines: recent developments, ongoing challenges and current candidates. Expert Review of Vaccines 12(8):933-53.

15. Guo C, Zhou Z, Wen Z, Liu Y, Zeng C, Xiao D, Ou M, Han Y, Huang S, Liu D, Ye X (2017). Global epidemiology of dengue outbreaks in 1990-2015: a systematic review and meta-analysis. Frontiers in Cellular and Infection Microbiology 7:317.

16. Patterson J, Sammon M, Garg M (2016). Dengue, Zika and chikungunya: emerging arboviruses in the New World. Western Journal of Emergency Medicine 17(6):671.

17. Salles TS, da Encarnação Sá-Guimarães T, de Alvarenga ES, Guimarães-Ribeiro V, de Meneses MD, de Castro-Salles PF, dos Santos CR, do Amaral Melo AC, Soares MR, Ferreira DF, Moreira MF (2018). History, epidemiology and diagnostics of dengue in the American and Brazilian contexts: a review. Parasites \& vectors 11(1):264.

18. Paixão ES, Teixeira MG, Rodrigues LC (2018). Zika, chikungunya and dengue: the causes and threats of new and re-emerging arboviral diseases. BMJ Global Health 3(Suppl 1):e000530. 